OPEN ACCESS

Edited by:

Valentina Pucino,

University of Birmingham,

United Kingdom

Reviewed by:

Steven O'Reilly,

Durham University, United Kingdom

Alessandra Pontillo,

University of São Paulo, Brazil

*Correspondence:

Marco Matucci-Cerinic

marco.matuccicerinic@unifi.it

Specialty section:

This article was submitted to

Autoimmune and

Autoinflammatory Disorders,

a section of the journal

Frontiers in Immunology

Received: 15 January 2021

Accepted: 02 March 2021

Published: 23 March 2021

Citation:

De Luca G, Cavalli G, Campochiaro C,

Bruni $C$, Tomelleri A, Dagna $L$ and Matucci-Cerinic M (2021) Interleukin-1 and Systemic Sclerosis: Getting to the

Heart of Cardiac Involvement.

Front. Immunol. 12:653950.

doi: 10.3389/fimmu.2021.653950

\section{Interleukin-1 and Systemic Sclerosis: Getting to the Heart of Cardiac Involvement}

\author{
Giacomo De Luca ${ }^{1,2}$, Giulio Cavalli ${ }^{1,2}$, Corrado Campochiaro ${ }^{1,2}$, Cosimo Bruni ${ }^{3}$, \\ Alessandro Tomelleri $^{1,2}$, Lorenzo Dagna ${ }^{1,2}$ and Marco Matucci-Cerinic ${ }^{3 *}$ \\ 1 Unit of Immunology, Rheumatology, Allergy and Rare diseases (UnIRAR), IRCCS San Raffaele Hospital, Milan, Italy, \\ 2 Vita-Salute San Raffaele University, Milan, Italy, ${ }^{3}$ Department of Experimental and Clinical Medicine, University of Florence, \\ and Division of Rheumatology AOUC, Florence, Italy
}

Systemic sclerosis (SSc) is rare, severe connective tissue disease characterized by endothelial and vascular damage, immune activation, and resulting in inflammation and fibrosis of skin and internal organs, including the heart. SSc is associated with high morbidity and mortality. Cardiac involvement is frequent in SSc patients, even though often asymptomatic at early stages, and represents one of the major causes of SScrelated mortality. Heart involvement has a variable clinical presentation, and its pathogenesis is not completely understood. Myocardial fibrosis is traditionally considered the immunopathologic hallmark of heart involvement in SSc. This unique histological feature is paralleled by distinctive clinical and prognostic features. The socalled "vascular hypothesis" represents the most credited hypothesis to explain myocardial fibrosis. More recently, the prominent role of an inflammatory myocardial process has been identified as a cardinal event in the evolution to fibrosis, thus also delineating an "inflammation-driven pathway to fibrosis". The pro-inflammatory cytokine interleukin (IL)-1 has an apical and cardinal role in the myocardial inflammatory cascade and in cardiac dysfunction. The primary aim of this perspective article is: to present the emerging evidence on the role of $\mathrm{IL}-1$ and inflammasome in both SSc and heart inflammation, to review the complex interplay between cellular metabolism and inflammasome activation, and to discuss the rationale for targeted inhibition of IL-1 for the treatment of SSc-heart involvement, providing preliminary experimental and clinical data to support this hypothesis.

Keywords: systemic sclerosis (scleroderma), heart inflammation, interleukin-1, inflammasome, cellular metabolism

\section{INTRODUCTION}

Heart involvement is frequent and is a major cause of mortality in systemic sclerosis (SSc), being responsible for up to $30 \%$ of disease-related deaths (1-3). Heart involvement has a variable clinical presentation: at early stages most patients are asymptomatic, but some go on to develop arrhythmias, dyspnea, chest pain, and heart failure (HF) (1, 4-9). In comparison to other 
inflammatory myocardial disease, myocardial fibrosis is usually considered the immunopathologic hallmark of SSc heart disease. This unique histological feature is paralleled by distinctive clinical and prognostic features $(10,11)$.

The most credited hypothesis to explain myocardial fibrosis is the one attributed to "vascular" (12): intermittent vascular spasm, ischemic necrosis, and reperfusion injury are considered pivotal mechanisms in fibrogenesis. More recently, the prominent role of an inflammatory myocardial process, clinically identified as a myocarditis, has been also identified as a crucial event in the evolution to fibrosis, thus also delineating an "inflammation-driven pathway to fibrosis" (1, 4, 13-18). In this "bimodal" ischemic-inflammatory pathogenic model, reperfusion products and pro-inflammatory cytokines may jointly orchestrate SSc-related heart involvement (SSc-HI). Therefore, the fact that only the inflammatory pathway to fibrosis is similar to other inflammatory cardiomyopathies ( $\mathrm{HF}$, dilated cardiomyopathy [DCM], virus-negative myocarditis) makes myocardial involvement in SSc a really peculiar and complex multifaceted event (19-24).

The pro-inflammatory cytokine interleukin (IL)-1 has an apical and cardinal role in the myocardial inflammatory cascade and in cardiac dysfunction (24). In this article, we provide an expert perspective on the emerging evidence on the role of IL-1 in both SSc and heart inflammation, and discuss the rationale for targeted inhibition of this cytokine for the treatment of SSc-HI.

\section{IL-1 FAMILY AND IL-1 BIOLOGY}

The IL-1 cytokines family includes seven members with agonistic

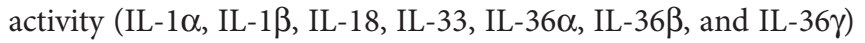
and four members with antagonistic functions (IL-1Ra, IL-36R $\alpha$, IL-37, and IL-38) (25-28).

IL-1 is an archetypal pro-inflammatory cytokine. The term IL- 1 hints at two different molecules, IL- $1 \alpha$ and IL- $1 \beta$, which share a significant sequence homology and bind the same IL-1 type-I receptor (IL-1RI), which then transduces pro-inflammatory signals and leads to the synthesis and expression of myriad secondary inflammatory mediators (29). IL- $1 \alpha$ is constitutively present in epithelial cells as a fully active pro-inflammatory mediator, and is released upon cell death thus acting as an "alarmin". Alarmins are a group of intracellular mediators, of which High Mobility Group Box 1 (HMGB1) likely represent the best characterized member, signaling tissue damage and activating inflammatory patrolling when found in the extracellular space. In scleroderma, mechanisms leading to alarmin release include ischemic cell death or inflammation-mediated tissue damage (30). Conversely, IL-1 $\beta$ is primarily produced by myeloid cells as an inactive precursor. Production of the mature proinflammatory cytokine follows activating cleavage of the precursor by an intracellular molecular complexes termed "inflammasomes" (31). To dampen excessive inflammation, the same cells that produce IL- $1 \alpha$ or IL- $1 \beta$ also synthesize diverse regulatory molecules, including the IL-1 receptor antagonist (IL$1 \mathrm{Ra})$. IL-1 signaling and IL-1-mediated inflammation are prevented by competitive binding of IL-1Ra to IL-1RI (30-33), thus curbing IL-1-mediated inflammation.

\section{IL-1 AND THE INFLAMMASOME IN SSC}

Expression or biologic activity of most IL-1 family cytokines can be abnormal in many autoimmune diseases, including SSc (34). IL- $1 \alpha$ regulates differentiation of fibroblast into myofibroblast, as well as myofibroblast longevity, which are considered central events in SSc (34). Indeed, dermal fibroblasts from SSc patients cultured ex vivo express higher levels of intracellular IL- $1 \alpha$ than healthy counterparts (35). Immunohistochemical studies indicated that intracellular IL- $1 \alpha$ is also markedly expressed in fibroblasts isolated from skin lesions of SSc patients; in addition, endogenous IL- $1 \alpha$ induces fibroblast proliferation and production of collagen by inducing IL- 6 and platelet-derived growth factor (PDGF) (36). Consistently, the production of IL-6, suppression of IL- $1 \alpha$ through IL- $1 \alpha$ siRNA results in decreased PDGF and procollagen production in SSc-affected fibroblasts (37), whereas overexpression of IL- $1 \alpha$ through transfection in healthy fibroblasts promotes differentiation into a SSc-related phenotype (34). In SSc fibroblasts, the NLRP3 inflammasome is over-expressed and caspase- 1 activity is up-regulated with consequent increased production of IL- $1 \beta$ and IL-18, whereas inhibition caspase- 1 and inflammasome activity abrogated the myofibroblast phenotype in SSc dermal and lung fibroblasts (3840). A separate study revealed that SSc fibroblasts exhibit increased synthesis of micro-RNA (miR)-155, which can also be induced by IL-1 $\beta$ (22). To date, miR-155 was implicated in various biological processes, including inflammation, immunity, and fibrosis (41). MiR-155, moreover, has been involved in cardiac remodeling, and miR155 deletion or inhibition reduced inflammatory and fibrotic responses in animal models of cardiac fibrosis induced by angiotensin-II (Ang-II) or diabetes $(42,43)$. In addition, miR-155 is required for the synthesis of collagen induced by activation of the inflammasome. Indeed, inhibition of caspase-1 activity abrogated miR-155 expression and significantly dampened collagen synthesis in a bleomycininduced SSc mouse model (22).

In SSc patients, high levels of IL-1 $\beta$ can be observed both in the bronchoalveolar lavage fluid (BAL) and in the serum (44). In the affected skin of SSc patients, IL-1 $\beta$ and IL-18 were significantly over-expressed, a finding correlating with the area of skin fibrosis assessed by the modified Rodnan skin score (mRSS) (38). This finding is not surprising, since IL-1 $\beta$ also induces myofibroblast activation, endothelial to mesenchymal transition, and fibrosis through IL-6 and TGF-1 $\beta$ (45).

IL- $1 \alpha$ is an intracellular cytokine which is rarely if ever detectable in the circulation, including in SSc patients (35). Similarly, even though associations between genes encoding IL-1 family cytokines and SSc susceptibility were revealed by genome-wide association studies, the results are not conclusive $(46-51)$. 


\section{INFLAMMASOME, IL-1, AND METABOLISM}

Recent studies have identified a strong interplay between cellular metabolism and inflammasome activation (52). Specifically, NLRP3 inflammasome is regulated by cellular metabolism, and growing evidences suggest that cellular metabolism is a crucial driver for macrophage polarization and inflammation, as well as myofibroblast differentiation and fibrosis $(53,54)$.

There are several molecular pathways involved in the metabolic regulation of the inflammasome: glycolysis, tricarboxylil acid (TCA) cycle, amino-acid metabolism, and fatty acid metabolism, and and most of them have been found to be dysregulated in SSc, providing a potential mechanism involved in inflammasome activation, and thus IL- $1 \beta$ release (52) (Figure 1).

Enhanced glycolisis is a hallmark of activated macrophages (53-57). Recents studies of SSc patients undergoing positron emission tomography using the glucose analogue tracer ${ }^{18}$ fluorodeoxyglucose revealed both increased glucose uptake (58). Glycolysis is critical in fibroblast differentiation and has been associated with the development of pulmonary fibrosis in bleomycin-induced experimental models (52). A recent study indicated that TGF- $\beta 1$, a key cytokine in scleroderma, upregulates glycolysis in dermal fibroblasts derived from SSc patients, and inhibition of glycolysis attenuates its pro-fibrotic effects (59).

Glutaminolysis through the TCA cycle and its intermediate metabolites was also evaluated in fibrotic conditions. TCA intermediate succinate binds the G-protein-coupled receptor-91 (GPR91) and increases GPR91, type-I collagen, $\alpha$-SMA, and TGF$\beta$ levels. Levels of succinate are up-regulated in lung myofibroblasts of patients with idiopathic pulmonary fibrosis, where they induce TGF- $\beta 1$, hypoxia-inducible factor-1alpha (HIF-1a), and fibroblast differentiation (58). Of note, succinate levels stabilize HIF-1a and promote IL-1 $\beta$ expression (53); this process is inhibited by itaconate, an anti-inflammatory metabolite required for the activation of the anti-inflammatory transcription factor Nrf2 by lipopolysaccharide in mouse and human macrophages, thus enabling Nrf2 to increase the expression of downstream antioxidant genes as $\mathrm{NAD}(\mathrm{P}) \mathrm{H}$ Quinone Dehydrogenase $1(60,61)$. Interestingly though, the Nrf2 pathway is highly down-regulated in human and SSc mice with detrimental consequences on inflammation and fibrosis. The $n r f 2^{-/-}$mice, indeed, develop a more severe SSc with enhanced fibrosis and inflammation compared to wild-type mice (62).

Results from the aformentioned study about the role of metabolic reprogramming in SSc pathogenesis (59), demonstrated that TGF- $\beta 1$ is able to enhance succinate production, which determines an increase of collagen expression, thus providing a link between the pro-fibrotic milieu

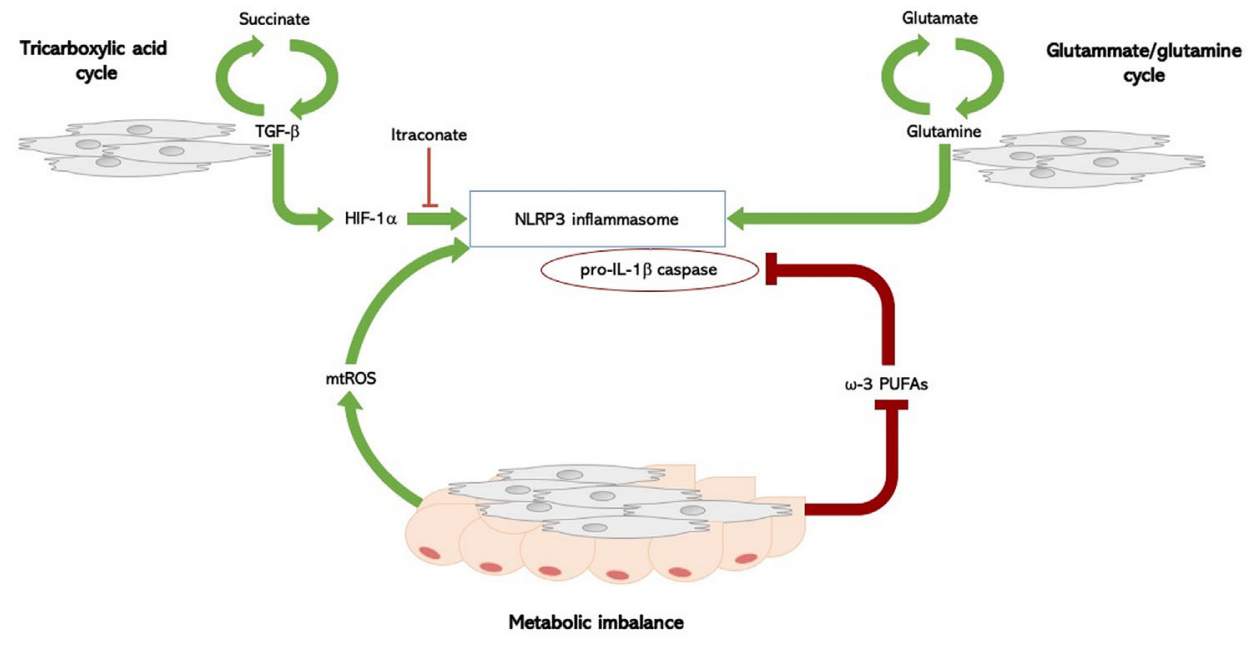

FIGURE 1 | Inflammasome and metabolism in systemic sclerosis. Molecular pathways involved in the metabolic regulation of the NLRP3 inflammasome in SSc: TCA cycle, fatty acid imbalance, and amino-acid metabolism. In SSc, a complex biological loop in which the TGF- $\beta 1$ rich microenvironment, the upregulated glutamine metabolism, and the fatty acid dysregulation, could lead to both inflammasome activation with IL-1 $\beta$ release and myofibroblasts differentiation, thus foraging the inflammation-driven fibrosis. Succinate is formed in the TCA cycle; its levels increase the TGF- $\beta 1$-induced HIF- $1 \alpha$ expression, promoting fibroblast differentiation. High levels of succinate can support IL- $1 \beta$ expression by stabilizing HIF- $1 \alpha$ for IL-1 $\beta$ transcript expression to occur. This process is inhibited by itaconate. TGF- $\beta 1$ itself is able to enhance succinate production, thus foraging this biological loop. SSc fibroblasts have an increased glutaminase expression, and an altered glutamine metabolism is an ubiquitous trait in SSc. The glutammate-glutamine pathway activates the NLPR3 inflammasome. Fatty acid metabolism has been implicated in the regulation of NLRP3 inflammasome: metabolic imbalance itself act as a cue to activate an inflammatory response, though the production of mitochondrial reactive oxygen species (mtROS), which directly activate the NLRP3 inflammasome. PUFAs, particularly $\omega$-3 PUFA, regulate NLRP3 inflammasome activation, acting as potent inhibitors of both caspase- 1 activation and IL-1 $\beta$ release. Fatty acid metabolism is dysregulated in SSc, and intradermal adipose tissue is atrophied and replaced by collagen-rich fibrous tissue in SSc. SSc, systemic sclerosis; TGF- $\beta 1$, transforming-growth factor beta- 1 ; HIF- $1 \alpha$, hypoxia-inducible factor-1alpha; TCA, tricarboxylil acid; IL-1 $\beta$, interleukin-1 beta; PUFAs, long-chain polyunsaturated fatty acids. 
of the disease and the metabolic activation of the inflammasome. Consistently, SSc fibroblasts incubated with itaconate exhibited reduced expression of collagen (59).

The same study showed that inhibition of glutamine metabolism, another pivotal metabolic pathway fuelling cellular growth, inflammation, and myofibroblast differentiation, antagonises TGF- $\beta 1$-induced glycolysis and fibrosis in normal human dermal fibroblasts. Furthermore, SSc fibroblasts showed an increase in glutaminase expression, suggesting that an altered glutamine metabolism may be a hallmark metabolic feature in SSc (59). Also of note, the same glutammate-glutamine pathway has been shown to activate the NLPR3 inflammasome (63).

Finally, fatty acid metabolism might also be implicated in the regulation of NLRP3 inflammasome. However, current evidence is conflicting and synthesis and degradation of fatty acids were linked to inflammasome activation in different studies, perhaps indicating that imbalance itself may activate an inflammatory response. It is also possible that these metabolic pathways activate a common intermediate mediator able to directly activate the NLRP3 inflammasome, the main candidate being mitochondrial reactive oxygen species (mtROS) (53). The $\omega-3$ PUFA, docosahexaenoic acid (DHA), inhibits the activation of caspase-1, thus lowering the production of active IL-1 $\beta(64,65)$. Apart from DHA, other $\omega-3$ PUFAs, such as eicosapentaenoic acid and $\alpha$-linolenic acid, can inhibit the activation of the inflammasome $(64,65)$.

The notion the fatty acid metabolism is dysregulated in SSc dates back to the 1970s, by studies showing that intradermal adipose tissue is progressively replaced by fibrotic tissue in SSc $(52,66)$.

Taken together, these findings support the existence of a complex biologic loop in SSc, in which the TGF- $\beta 1$ rich microenvironment, the up-regulated glutamine metabolism, the ehnahced glycolysis, and the fatty acid dysregulation, could all contribute to both inflammasome activation with IL-1 $\beta$ release and myofibroblasts differentiation, thus possibly foraging the occurrence of inflammation-driven fibrosis.

\section{IL-1 AND HEART INFLAMMATION}

Recent clinical and experimental data support the relevance of IL-1 in heart inflammation and cardiac dysfunction in several heart diseases. The heart exhibits a highly conserved response to tissue damage, characterized by a stereotyped inflammatory reaction that is centrally mediated by the pro-inflammatory cytokine IL-1 (24). Specifically, IL-1 $\alpha$ is released from the dying myocardial cells together with and other intracellular contents, which act as mediators activating the inflammasomes in bystander cells $(29,32,33,67-69)$. IL-1-mediated inflammation ensues; if protracted, this leads to the apoptosis of cardiomyocytes and to the loss of contractile tissue progressively replaced by fibrosis, clinically manifested with cardiomyopathy, HF, and arrhythmic outburst (24).

Previous studies evaluating endomyocardial biopsy (EMB) samples from patients with acute lymphocytic myocarditis indicated that intracellular aggregates of either Apoptosisassociated speck-like protein containing CARD (ASC) or caspase-1, both indicative of inflammasome activation, can cardiomyocytes and infiltrating immune cells. Notably, the number of inflammasome-containing leukocytes correlated with the clinical severity of HF (67).

Moreover, IL-1 causes impaired contractile function by inducing multiple downstream events, including uncoupling of the $\beta$-adrenergic receptor from the adenylyl cyclase, inhibition of L-type calcium channels $(24,28,67-74)$, transcriptional and posttranslational changes in phospholamban and sarcoplasmic/ endoplasmic reticulum calcium ATPase $(24,75)$, mytochondrial dysfunction, and nitric oxide (NO) synthesis (24, 76-78). Animal studies also confirm a role of IL-1-mediated inflammation in HF: injection of plasma from $\mathrm{HF}$ patients induced contractile dysfunction in mice, suggesting the existence of cardiodepressant factors in the circulation (79-82). Notably, administration of IL-1 $\beta$ to mice had similar effects, whereas pre-administration of IL-1 inhibitors prevented contractile dysfunction induced by HF serum: collectively considered, these findings indicate that cardiodepressant effects are centrally mediated by IL-1 (75-77), as also in sepsis (82).

Robust evidence also indicates that IL-1 signaling is central to the development of inflammation in both viral and autoimmune acute myocarditis (AMy). Mouse models of coxsackievirusinduced myocarditis exhibit heart infiltration with myeloid cells secreting IL- 1 and TNF- $\alpha$ (83). Increased IL- $1 \beta$ expression is also a feature of chronic heart inflammation in experimental models of post-myocarditis DCM, induced by infection with encephalomyocarditis virus (84). Mice lacking IL-1RI did not develop AMy (85), and administration or overexpression of IL-1Ra reduced disease severity in experimental models of cardiomyopathy (86-89). These pre-clinical findings were paralleled by clinical observations in humans: EMBs from patients with viral myocarditis (85) and idiopathic DCM (86) revealed increased IL-1 $\beta$ mRNA levels.

\section{HEART INFLAMMATION DOWNSTREAM IL-1}

Once induced, inflammation escalates into a redundant process: hence, other pro-inflammatory cytokines may also play a key role in heart inflammation and inflammation-driven fibrosis. The IL-1 biological activity sustains an inflammatory process which involves IL-1 itself as well as downstream mediators. IL-6 is induced by IL-1, and acts as a downstream mediator of several inflammatory effects (Figure 2). It is thereby not surprising that IL-6 concentrations are elevated in the serum and myocardium of patients with $\mathrm{HF}$ and myocarditis, while also being predictive of adverse outcomes (90). In myocarditis, the primary sources of IL-6 are likely cardiomyocytes and cardiac fibroblasts (91, 92). Overexpression of IL-6 in experimental animals subjected to viral myocarditis results in extensive myocardial inflammation, whereas IL-6 inhibition with tocilizumab reduced heart inflammation and infiltration with CD3+T-cells and CD68+ macrophages (20). 


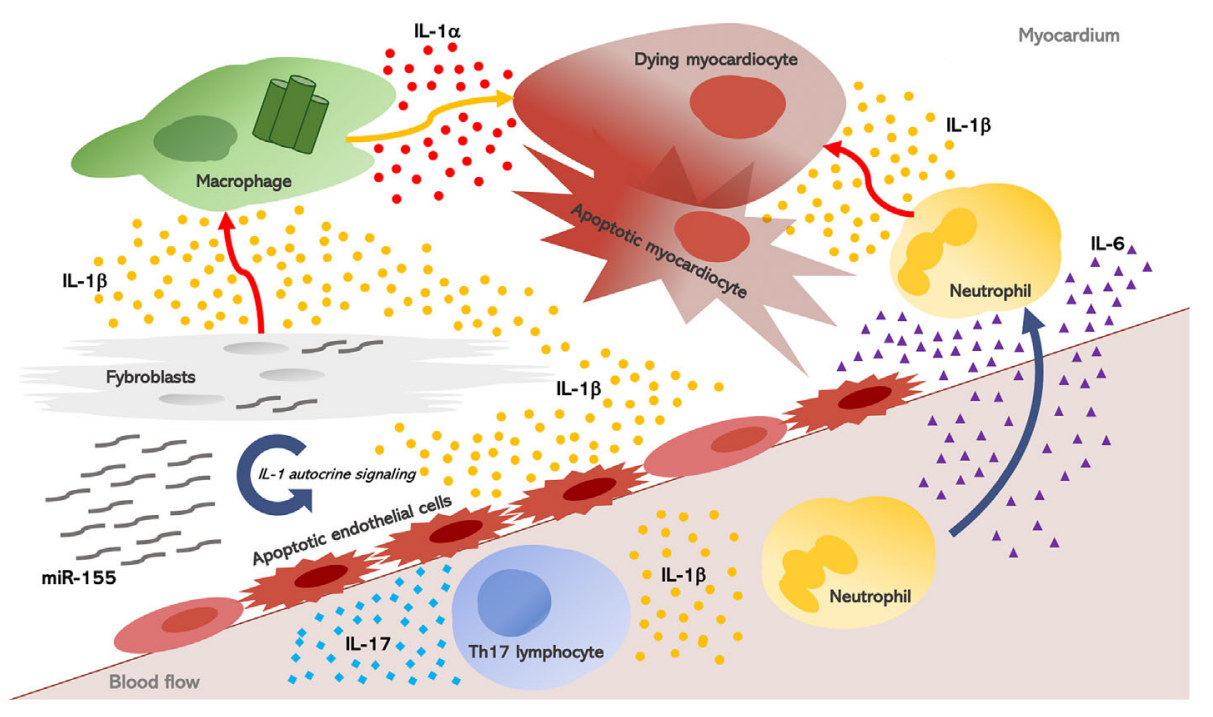

FIGURE 2 | Interleukin-1, myocardial inflammation, and heart fibrosis in systemic sclerosis. Heart inflammation results in myocardial injury. As a consequence, IL-1 $\alpha$ is released from dying myocardiocytes, together with intracellular debris and inflammatory mediators; these in turn activate a molecular complex known as the "inflammasome" inside macrophages which processes and releases active IL-1 $\beta$. Once induced, inflammation escalates into a redundant process: hence, other proinflammatory cytokines, mainly IL-6, are produced and they perpetuate heart inflammation and inflammation-driven fibrosis. IL-1 and IL-6 also promote Th17 differentiation, and in post-myocarditis, the role of IL-17A emerged in myocardiac remodeling, thus contributing to both myocardial fibrosis and progression to dilated cardiomyopathy. Finally, in SSc fibroblasts, the NLRP3 inflammasome is over-expressed with consequent increased production of IL-1b. IL-1 also stimulate SSc fibroblasts and induce the synthesis of micro-RNA (miR)-155 which establishes an autocrine loop further increasing IL-1 signaling.

Recently, tocilizumab was used to effectively treat SSc-related myocarditis, and improvement of myocardial inflammation was revealed as a reduction in myocardial edema at cardiac magnetic resonance (CMR), and by the improvement of cardiac function, clinical status and cardiac enzymes (20). Moreover, IL-6 plays a major role in heart fibrosis induced by Ang-II, through TGF- $\beta$ / Smad activation. Consistently, IL-6 deficiency reduces cardiac inflammation, as well as contractile dysfunction and interstitial fibrosis, without affecting blood pressure in Ang-II-high saltinduced hypertension in IL-6 knockout (IL-6-/-) mice (21). Furthermore, deletion of IL- 6 alleviates interstitial fibrosis also in experimental diabetic cardiomyopathy in IL-6-/- mice (81). The deletion or inhibition of miR155 yielded the same protective effects $(42,43)$, and recent studies revealed that the soluble IL-6 receptor (IL6R) is a target of miR155 (93). In summary, these studies delineate a miR-155/IL-1/IL-6 loop sustaining inflammation-driven fibrosis: overexpression of miR-155 in SSc fibroblasts induces inflammasome-mediated release of IL-1 $\beta$, which in turn stimulates IL-6 production and collagen synthesis during fibrosis.

Another important signaling axis which potentially contributes to fibrosis and inflammation in SSc is the IL-1/IL17 axis. Many of inflammatory cytokines that are involved in the SSc pathogenesis, (i.e., IL-1, IL-6, TGF- $\beta$ ), also promote Th17 differentiation. This strongly suggests their potential role in skewing CD4+ T cells toward Th17 differentiation in SSc. A recent in vivo study showed that IL-17 is involved in fibrosis and inflammation in bleomycin (BLM)-induced SSc. The authors also used another murine model of SSc, chronic graft-versus-host disease(cGVHD), to show that blocking IL-17 activity was able to attenuate disease severity. IL-1 and IL-17 synergically induce the expression of profibrotic and inflammatory mediators, both in human and murine dermal fibroblasts. Subsequent animal studies in vivo confirmed the antifibrotic and antiinflammatory potential of IL-1Ra (94). Hence, IL-17 inhibition, either directly or by blocking IL-1, has therapeutic rationale for tissue fibrosis in SSc. In post-myocarditis, the role of IL-17A emerged either in myocardiac remodeling and the progression to DCM, thus contributing to myocardial fibrosis following experimental AMy by a protein-kinase-C(PKC)b/Erk1/2 Nuclear Factor (NF)-kB signaling $(95,96)$.

\section{THERAPEUTIC APPLICABILITY OF IL-1 INHIBITION AND FUTURE PERSPECTIVES}

Despite extensive experimental evidence pointing at a central role for IL-1 in the pathogenesis of heart inflammation, systolic dysfunction, and fibrosis, and despite a possible role of this cytokine in SSc skin and lung inflammation, the use of available IL-1 blocking agents in SSc was only anedoctally reported.

Rilonacept, a fusion protein consisting of the human IL-1 receptor (IL-1R1) and IL-1 receptor accessory protein (IL$1 \mathrm{RAcP}$ ) which binds and neutralizes both IL- $\alpha$ and $-\beta$, was evaluated in single phase I/II randomized, double-blind, placebocontrolled trial on SSc patients. The primary endpoint was the 
level of skin expression of the 2G SSc gene biomarkers, which functions as a proxy for the mRSS, while the secondary endpoint was the change in mRSS. Nineteen patients were randomized 2:1 rilonacept $320 \mathrm{mg}$ loading dose at day 0 and then $160 \mathrm{mg}$ weekly versus placebo. Skin biopsies were obtained before rilonacept treatment initiation and at week 7 . Both the primary and the secondary endpoints were not met, as no changes in gene expression or in the mRSS between treated and placebo patients were observed after 6 weeks (97). However, this trial had several limitations, including the small sample size and the short duration of therapy, even more important in the context of a chronic fibrotic disease. Moreover, no exploratory secondary endpoints to evaluate SSc-HI were considered.

Data from animal models are scarce and conflicting. Treatment with anakinra, a recombinant IL-1 receptor antagonist, improved BLM-induced pulmonary fibrosis in mice and of pulmonary silicosis in humans $(98,99)$, as well as pulmonary function in patients with COVID-19 and systemic hyperinflammation (100). However, anakinra aggravated pulmonary fibrosis due to Th2 skewing in the fos-related antigen-2 (Fra-2) mouse model of SSc, and was not associated to changes in lung inflammation profile in wild type mice (101). To date, the Fra-2 transgenic mice spontaneously develop pulmonary inflammation. These findings on animal models suggest that the net effect of IL-1 (or of its inhibition) is context-dependent. For this reason, it is still premature to transfer these limited data, obtained on animal models, into clinical practice.

It is important to note that recent evidence supports IL-1 therapeutic blockade in HF and myocarditis. In fact, given the role of IL-1 in both heart inflammation and contractile dysfunction, and the potential role in mechanisms of inflammation-driven heart fibrosis through different pathways, IL-1 inhibition might fit in a proof of concept rationale for an anti-inflammatory therapeutic strategy in SSc myocarditis. In patients with myocardial inflammation and HF, the short-term treatment regimen (14 days) with anakinra improved exercise capability, as determined by oxygen consumption $\left(\mathrm{VO}_{2}\right)(79,102)$. Prolonged administration (12 weeks) reduced hospitalizations and further improved $\mathrm{VO}_{2}$, NTproBNP, and the quality of life in a randomized clinical trial (RCT) (103). Similarly, treatment with the anti-IL-1 $\beta$ monoclonal-antibody canakinumab significantly reduced cardiovascular events in 10,061 patients with previous myocardial infarction and C-reacrive protein $>2 \mathrm{mg} / \mathrm{L}$ in the CANTOS-trial (104). Recently, our group firstly described the dramatic dampening of heart inflammation and an unprecedented clinical improvement after IL-1 suppression with anakinra in a patient with DCM. Improvement began soon after anakinra administration with improvement of the arrhythmic outburst, decrease of cardiac biomarkers, and normalized echocardiography and CMR imaging-including those that measured LVEF (105). Anakinra treatment was also beneficial in patients with fulminant myocarditis (106-108). Taken together, these observations suggest that IL-1 inhibition could curb heart inflammation while also ameliorating myocardial contractility. Thus, IL-1 inhibition may dampen disease progression and fibrotic damage in patients with myocarditis and other inflammatory cardiomyopathies.

A significant bulk of data also demonstrates the efficacy of IL-1 blockade in pericardial inflammation and recurrent pericarditis (109-113), which usually requires steroids and immunosuppressive treatment when clinically evident (1), including a RCT of anakinra in 21 patients (96), and more recently a positive trial with rilonacept (114). This is of great importance since pericarditis is common in SSc: symptomatic pericarditis occurs in $5-16 \%$ patients, whereas autopsydemonstrated pericardial involvement occurs in $33-72 \%$ patients (1).

Beside the aforementioned RCTs in $\operatorname{HF}(79,102,103)$, double blind, phase IIb, randomized, placebo-controlled clinical trials of anakinra are ongoing to evaluate this treatment in acute myocarditis [ARAMIS-trial, ClinicalTrials.gov: Identifier: NCT03018834] and EMB-proven virus-negative myocarditis [MYTH-1 trial; Eudract: 2018-003472-13]. The monoclonal antibody canakinumab is also clinically available, which blocks IL-1 $\beta 111)$. More recently, oral NLRP3 inflammasome inhibitors have been proposed to treat a wide spectrum of inflammatory cardiovascular diseases, and RCTs are ongoing (115).

Results from these trials, together with the robust biologic proof of concept, could potentially pave the way to the use of IL-1 therapeutic blockade to treat SSc-related inflammatory heart involvement.

\section{DATA AVAILABILITY STATEMENT}

The original contributions presented in the study are included in the article/supplementary material. Further inquiries can be directed to the corresponding author.

\section{AUTHOR CONTRIBUTIONS}

GDL: conceived the hypothesis, contributed to the understanding of pathogenic mechanisms of both systemic sclerosis heart involvement and IL-1 mediated heart inflammation, generated original data to support the hypothesis, and drafted the manuscript. GC: contributed to the understanding of biological effects of IL-1 and IL-1 therapeutic blockade in a broad spectrum of rheumatic and inflammatory diseases, generated original data to support the hypothesis, and critically revised the manuscript. CC: contributed to the understanding of pathogenic mechanisms of both systemic sclerosis heart involvement and IL-1 mediated heart inflammation, and critically revised the manuscript. CB: contributed to the understanding of pathogenic mechanisms, clinical presentation, and prognostic meaning of systemic sclerosis, particularly heart involvement, and critically revised the manuscript. AT: contributed to the understanding of biological effects of IL-1 and IL-1 therapeutic blockade in a broad spectrum of rheumatic and inflammatory diseases, created 
the figures, and critically revised the manuscript. LD: conceived the hypothesis, critically revised the manuscript, and gave the approval of the final version. MM-C: conceived the hypothesis,

\section{REFERENCES}

1. Champion HC. The heart in scleroderma. Rheum Dis Clin North Am (2008) 34:181-90;viii. doi: 10.1016/j.rdc.2007.12.002

2. Tyndall JA, Bannert B, Vonk M, Airò P, Cozzi F, Carreira PE, et al. Causes and risk factors for death in systemic sclerosis: a study from the Systemic sclerosis myocarditis EULAR Scleroderma Trials and Research (EUSTAR) database. Ann Rheum Dis (2010) 69:1809-15. doi: 10.1136/ard.2009. 114264

3. Bournia VK, Tountas C, Protogerou AD, Panopoulos S, Mavrogeni S, Sfikakis PP, et al. Update on assessment and management of primary cardiac involvement in systemic sclerosis. J scleroderma Relat Disord (2018) 3(1):53-65. doi: 10.1177/2397198317747441

4. Pieroni M, De Santis M, Zizzo G, Bosello S, Smaldone C, Campioni M, et al. Recognizing and treating myocarditis in recent-onset systemic sclerosis heart disease: potential utility of immunosuppressive therapy in cardiac damage progression. Semin Arthritis Rheum (2014) 43:526-35. doi: 10.1016/ j.semarthrit.2013.07.006

5. De Luca G, Bosello SL, Gabrielli FA, Berardi G, Parisi F, Rucco M, et al. Prognostic Role of Ventricular Ectopic Beats in Systemic Sclerosis: A Prospective Cohort Study Shows ECG Indexes Predicting the Worse Outcome. PloS One (2016) Apr 2111(4):e0153012. doi: 10.1371/ journal.pone. 0153012

6. Bernardo P, Conforti ML, Bellando-Randone S, Pieragnoli P, Blagojevic J, Kaloudi $\mathrm{O}$, et al. Implantable cardioverter defibrillator prevents sudden death in systemic sclerosis. J Rheumatol (2011) 38:1617-21. doi: 10.3899/ jrheum. 100480

7. Peretto G, Sala S, Basso C, Rizzo S, Radinovic A, Frontera A, et al. Inflammation as a Predictor of Recurrent Ventricular Tachycardia After Ablation in Patients With Myocarditis. J Am Coll Cardiol (2020) Oct 676 (14):1644-56. doi: 10.1016/j.jacc.2020.08.012

8. De Luca G, Bosello SL, Canestrari G, Cavalli G, Dagna L, Ferraccioli G. QTc interval prolongation in Systemic Sclerosis: Correlations with clinical variables and arrhythmic risk. Int J Cardiol (2017) Jul 15239:33. doi: 10.1016/j.ijcard.2017.03.088

9. Barsotti S, Stagnaro C, D'Ascanio C, Parma A, Emdin M, Conti U, et al. High sensitivity troponin might be a marker of subclinical scleroderma heart involvement: a preliminary study. J scleroderma Relat Disord (2017) 2 (3):183. doi: $10.5301 /$ jsrd.5000244

10. De Luca G, Campochiaro C, De Santis M, Sartorelli S, Peretto G, Sala S, et al. Systemic sclerosis myocarditis has unique clinical, histological and prognostic features: a comparative histological analysis. Rheumatol (Oxford) (2020) Sep 159(9):2523-33. doi: 10.1093/rheumatology/kez658

11. Lee DC, Hinchcliff ME, Sarnari R, Stark MM, Lee J, Koloms K, et al. Diffuse cardiac fibrosis quantification in early systemic sclerosis by magnetic resonance imaging and correlation with skin fibrosis. J scleroderma Relat Disord (2018) 3(2):159-69. doi: 10.1177/239719831876288

12. Bulkley BH, Ridolfi RL, Salyer WR, Hutchins GM. Myocardial lesions of progressive systemic sclerosis. A cause of cardiac dysfunction. Circulation. (1976) Mar53(3):483-90. doi: 10.1161/01.cir.53.3.483

13. De Luca G, Campochiaro C, Sartorelli S, Franchini S, Candela C, Peretto G, et al. Unexpected acute lymphocytic virus-negative myocarditis in a patient with limited cutaneous systemic sclerosis: a case report. Scand J Rheumatol (2018) 30:1-2. doi: 10.1080/03009742.2018.1493744

14. Bosello S, De Luca G, Ferraccioli G. Troponin in stable ischemic heart disease and diabetes. N Engl J Med (2015) 373:1977-8. doi: 10.1056/ NEJMoa1415921

15. De Luca G, Bosello S, Leone AM, Gabrielli F, Pelargonio G, Inzani F, et al. Life-threatening arrhythmias in a scleroderma patient: the role of myocardial inflammation in arrhythmic outburst. Scand J Rheumatol (2017) 46:78-80. doi: 10.3109/03009742.2016.1157626

16. De Luca G, Bosello S, Leone AM, Gabrielli F, Pelargonio G, Inzani F, et al. Life-threatening arrhythmias in a scleroderma patient: the role of myocardial critically revised the manuscript, and gave the approval of the final version. All authors contributed to the article and approved the submitted version.

inflammation in arrhythmic outburst. Scand J Rheumatol (2017) Jan46 (1):78-80. doi: $10.3109 / 03009742.2016 .1157626$

17. Peretto G, Sala S, De Luca G, Campochiaro C, Sartorelli S, Cappelletti AM, et al. Impact of systemic immune-mediated diseases on clinical features and prognosis of patients with biopsy-proved myocarditis. Int J Cardiol (2019) Apr 1280:110-6. doi: 10.1016/j.ijcard.2018.11.104

18. Mueller KA, Mueller II, Eppler D, Zuern CS, Seizer P, Kramer U, et al. Clinical and histopathological features of patients with systemic sclerosis undergoing endomyocardial biopsy. PloS One (2015) May 1210(5): e0126707. doi: 10.1371/journal.pone.0126707

19. Westermann D, Lindner D, Kasner M, Zietsch C, Savvatis K, Escher F, et al. Cardiac inflammation contributes to changes in the extracellular matrix in patients with heart failure and normal ejection fraction. Circ Heart Fail (2011) Jan4(1):44-52. doi: 10.1161/CIRCHEARTFAILURE.109.931451

20. Ma F, Li Y, Jia L, Han Y, Cheng J, Li H, et al. Macrophage-stimulated cardiac fibroblast production of IL- 6 is essential for TGF $\beta / S$ mad activation and cardiac fibrosis induced by angiotensin II. PloS One (2012) 7(5):e35144. doi: 10.1371/journal.pone.0035144

21. Zhang Y, Wang JH, Zhang YY, Wang YZ, Wang J, Zhao Y, et al. Deletion of interleukin-6 alleviated interstitial fibrosis in streptozotocin-induced diabetic cardiomyopathy of mice through affecting TGF $\beta 1$ and miR-29 pathways. Sci Rep (2016) Mar 146:23010. doi: 10.1038/srep23010

22. Artlett CM, Sassi-Gaha S, Hope JL, Feghali-Bostwick CA, Katsikis PD. Mir155 is overexpressed in systemic sclerosis fibroblasts and is required for NLRP3 inflammasome-mediated collagen synthesis during fibrosis. Arthritis Res Ther (2017) Jun 1719(1):144. doi: 10.1186/s13075-017-1331-z

23. Mitchell MD, Laird RE, Brown RD, Long CS. IL-1beta stimulates rat cardiac fibroblast migration via MAP kinase pathways. Am J Physiol Heart Circ Physiol (2007) Feb;292(2):H1139-47. doi: 10.1152/ajpheart.00881

24. De Luca G, Cavalli G, Campochiaro C, Tresoldi M, Dagna L. Myocarditis: An Interleukin-1-Mediated Disease? Front Immunol (2018) 13;9:1335. doi: $10.3389 /$ fimmu. 2018.01335

25. Dinarello C, Arend W, Sims J, Smith D, Blumberg H, O’Neill L, et al. IL-1 family nomenclature. Nat Immunol (2010) 11:973. doi: 10.1038/ni1110-973

26. Ballak DB, Li S, Cavalli G, Stahl JL, Tengesdal IW, van Diepen JA, et al. Interleukin-37 treatment of mice with metabolic syndrome improves insulin sensitivity and reduces pro-inflammatory cytokine production in adipose tissue. J Biol Chem (2018) Sep 14293(37):14224-36. doi: 10.1074/ jbc.RA118.003698

27. Klück V, van Deuren RC, Cavalli G, Shaukat A, Arts P, Cleophas MC, et al. Rare genetic variants in interleukin-37 link this anti-inflammatory cytokine to the pathogenesis and treatment of gout. Ann Rheum Dis (2020) Apr79 (4):536-44. doi: 10.1136/annrheumdis-2019-216233

28. Cavalli G, Justice JN, Boyle KE, D’Alessandro A, Eisenmesser EZ, Herrera JJ, et al. Interleukin 37 reverses the metabolic cost of inflammation, increases oxidative respiration, and improves exercise tolerance. Proc Natl Acad Sci U S A. (2017) Feb 28114(9):2313-8. doi: 10.1073/pnas.1619011114

29. Dinarello CA. Interleukin-1 in the pathogenesis and treatment of inflammatory diseases. Blood. (2011) Apr 7117(14):3720-32. doi: 10.1182/ blood-2010-07-273417

30. Cavalli G, Colafrancesco S, Emmi G, Imazio M, Lopalco G, Maggio MC, et al. Interleukin $1 \alpha$ : a comprehensive review on the role of IL-1 $\alpha$ in the pathogenesis and targeted treatment of autoimmune and inflammatory diseases. Autoimmun Rev (2021) 20(3):102763. doi: 10.1016/j.autrev. 2021.102763

31. Cavalli G, Cenci S. Autophagy and Protein Secretion. J Mol Biol (2020) 432 (8):2525-45. doi: 10.1016/j.jmb.2020.01.015

32. Bujak M, Frangogiannis NG. The role of IL-1 in the pathogenesis of heart disease. Arch Immunol Ther Exp (Warsz) (2009) May-Jun57(3):165-76. doi: 10.1007/s00005-009-0024-y

33. Cavalli G, Dinarello CA. Suppression of inflammation and acquired immunity by IL-37. Immunol Rev (2018) Jan281(1):179-90. doi: 10.1111/ imr.12605 
34. Xu D, Mu R, Wei X. The Roles of IL-1 Family Cytokines in the Pathogenesis of Systemic Sclerosis. Front Immunol (2019) Sep 1310:2025. doi: 10.3389/ fimmu.2019.02025

35. Higgins GC, Wu Y, Postlethwaite AE. Intracellular IL-1 receptor antagonist is elevated in human dermal fibroblasts that overexpress intracellular precursor IL-1 alpha. J Immunol (1999) Oct 1163(7):3969-75.

36. Kawaguchi Y, Hara M, Wright TM. Endogenous IL-1a fromsystemic sclerosis fibroblasts induces IL-6 and PDGF-A. J Clin Invest (1999) 103:1253-60. doi: 10.1172/JCI4304

37. Kawaguchi Y, McCarthy SA, Watkins SC, Wright TM. Autocrine activation by interleukin 1alpha induces the fibrogenic phenotype of systemic sclerosis fibroblasts. J Rheumatol (2004) Oct31(10):1946-54.

38. Martínez-Godínez MA, Cruz-Domínguez MP, Jara LJ, Domínguez-López A, Jarillo-Luna RA, Vera-Lastra O, et al. Expression of NLRP3 inflammasome, cytokines and vascular mediators in the skin of systemic sclerosis patients. Isr Med Assoc J (2015) Jan17(1):5-10.

39. Artlett CM, Sassi-Gaha S, Rieger JL, Boesteanu AC, Feghali-Bostwick CA, Katsikis PD. The inflammasome activating caspase 1 mediates fibrosis and myofibroblast differentiation in systemic sclerosis. Arthritis Rheumatol (2011) Nov63(11):3563-74. doi: 10.1002/art.30568

40. Henderson J, Bhattacharyya S, Varga J, O'Reilly S. Targeting TLRs and the inflammasome in systemic sclerosis. Pharmacol Ther (2018) Dec192:163-9. doi: 10.1016/j.pharmthera.2018.08.003

41. Chen C, Ponnusamy M, Liu C, Gao J, Wang K, Li P. MicroRNA as a Therapeutic Target in Cardiac Remodeling. BioMed Res Int (2017) 2017:1278436. doi: 10.1155/2017/1278436

42. Zhang D, Cui Y, Li B, Luo X, Li B, Tang Y. miR-155 regulates high glucoseinduced cardiac fibrosis via the TGF- $\beta$ signaling pathway. Mol Biosyst (2016) Dec 2013(1):215-24. doi: 10.1039/c6mb00649c

43. Wei Y, Yan X, Yan L, Hu F, Ma W, Wang Y, et al. Inhibition of microRNA-155 ameliorates cardiac fibrosis in the process of angiotensin II-induced cardiac remodeling. Mol Med Rep (2017) Nov16(5):7287-96. doi: 10.3892/mmr.2017.7584

44. Hussein MR, Hassan HI, Hofny ER, Elkholy M, Fatehy NA, Abd Elmoniem $\mathrm{AE}$, et al. Alterations of mononuclear inflammatory cells, CD4/CD8+ T cells, interleukin $1 \mathrm{~b}$, and tumour necrosis factor alpha in the bronchoalveolar lavage fluid, peripheral blood, and skin of patients with systemic sclerosis. J Clin Pathol (2005) 58:178-84. doi: 10.1136/jcp.2004.019224

45. Maleszewska M, Moonen JR, Huijkman N, van de Sluis B, Krenning G, Harmsen MC. IL-1b and TGFb2 synergistically induce endothelial to mesenchymal transition in an NFkappaB-dependent manner. Immunobiology. (2013) 218:443-54. doi: 10.1016/j.imbio.2012.05.026

46. Huang XL, Wu GC, Wang YJ, Yang XK, Yang GJ, Tao JH, et al. Association of interleukin-1 family cytokines single nucleotide polymorphisms with susceptibility to systemic sclerosis: an independent case-control study and a meta-analysis. Immunol Res (2016) 64:1041-52. doi: 10.1007/s12026-016-8797-7

47. Hutyrova B, Lukac J, Bosak V, Buc M, du Bois R, Petrek M. Interleukin 1a single-nucleotide polymorphism associated with systemic sclerosis. J Rheumatol (2004) 31:81-4.

48. Kawaguchi Y, Tochimoto A, Ichikawa N, Harigai M, Hara M, Kotake S, et al. Association of IL1A gene polymorphisms with susceptibility to and severity of systemic sclerosis in the Japanese population. Arthritis Rheumatol (2003) 48:186-92. doi: 10.1002/art.10736

49. Abtahi S, Farazmand A, Mahmoudi M, Ashraf-Ganjouei A, Javinani A, Nazari B, et al. IL-1A rs1800587, IL-1B rs1143634 and IL-1R1 rs2234650 polymorphisms in Iranian patients with systemic sclerosis. Int $J$ Immunogenet (2015) 42:423-7. doi: 10.1111/iji.12212

50. Su H, Rei N, Zhang L, Cheng J. Meta-analyses of IL1A polymorphisms and the risk of several autoimmune diseases published in databases. PloS One (2018) 13:e0198693. doi: 10.1371/journal.pone.0198693

51. Khazim K, Azulay EE, Kristal B, Cohen I. Interleukin 1 gene polymorphism and susceptibility to disease. Immunol Rev (2018) Jan281(1):40-56. doi: $10.1111 / \mathrm{imr} .12620$

52. Zhu H, Chen W, Liu D, Luo H. The role of metabolism in the pathogenesis of systemic sclerosis. Metabolism. (2019) Apr93:44-51. doi: 10.1016/ j.metabol.2018.12.004

53. Meyers AK, Zhu X. The NLRP3 Inflammasome: Metabolic Regulation and Contribution to Inflammaging. Cells. (2020) 9(8):1808. doi: 10.3390/ cells 9081808
54. Villa A, Belloni D, Vergani B, Cenci S, Cavalli G, Biavasco R, et al. 3D culture of Erdheim-Chester disease tissues unveils histiocyte metabolism as a new therapeutic target: Annals of the Rheumatic Diseases. Ann Rheum Dis (2018) 78(6):862-4. doi: 10.1136/annrheumdis-2018-214432

55. Cavalli G, De Luca G, Doglioni C, Ferrero E, Ferrarini M, Dagna L. A Novel Histiocytosis With Synovial and Skin Involvement. Ann Internal Med (2020). doi: 10.7326/L20-0092

56. Pacini G, Cavalli G, Tomelleri A, De Luca G, Pacini G, Ferrarini M, et al. The fibrogenic chemokine CCL18 is associated with disease severity in ErdheimChester disease. OncoImmunology (2018) 7(7):e1440929. doi: 10.1080/ 2162402X.2018.1440929

57. O'Neill LAJ, Kishton RJ. Rathmell J. A guide to immunometabolism for immunologists. Nat Rev Immunol (2016) 16:553-65. doi: 10.1038/ nri.2016.70

58. Nishiyama Y, Yamamoto $\mathrm{Y}$, Dobashi H. Clinical value of $18 \mathrm{~F}$ fluorodeoxyglucose positron emission tomography in patients with connective tissue disease. Jpn J Radiol (2010) 28:405-13. doi: 10.1007/ s11604-010-0445-x

59. Henderson J, Duffy L, Stratton R, Ford D, O’Reilly S. Metabolic reprogramming of glycolysis and glutamine metabolism are key events in myofibroblast transition in systemic sclerosis pathogenesis. J Cell Mol Med (2020) 24:14026-. doi: $10.1111 / \mathrm{jcmm} .16013$

60. Lampropoulou V, Sergushichev A, Bambouskova M, Nair S, Vincent EE, Loginicheva E, et al. Itaconate Links Inhibition of Succinate Dehydrogenase with Macrophage Metabolic Remodeling and Regulation of Inflammation. Cell Metab (2016) 24:158-66. doi: 10.1016/j.cmet.2016.06.004

61. Mills EL, Ryan DG, Prag HA, Dikovskaya D, Menon D, Zaslona Z, et al. Itaconate is an anti-inflammatory metabolite that activates $\mathrm{Nrf} 2$ via alkylation of KEAP1. Nature. (2018) Apr 5556(7699):113-7. doi: 10.1038/ nature25986

62. Kavian N, Mehlal S, Jeljeli M, Saidu NEB, Nicco C, Cerles O, et al. The Nrf2Antioxidant Response Element Signaling Pathway Controls Fibrosis and Autoimmunity in Scleroderma. Front Immunol (2018) 9:1896. doi: 10.3389/ fimmu.2018.01896

63. Yang SJ, Han AR, Kim EA, Yang JW, Ahn JY, Na JM, et al. KHG21834 attenuates glutamate-induced mitochondrial damage, apoptosis, and NLRP3 inflammasome activation in SH-SY5Y human neuroblastoma cells. Eur J Pharmacol (2019) 856:172412. doi: 10.1016/j.ejphar.2019.172412

64. Yan Y, Jiang W, Spinetti T, Tardivel A, Castillo R, Bourquin C, et al. Omega3 fatty acids prevent inflammation and metabolic disorder through inhibition of NLRP3 inflammasome activation. Immunity (2013) 38:115463. doi: 10.1016/j.immuni.2013.05.015

65. Williams-Bey Y, Boularan C, Vural A, Huang NN, Hwang IY, Shan-Shi C, et al. Omega-3 free fatty acids suppress macrophage inflammasome activation by inhibiting NF- $\mathrm{KB}$ activation and enhancing autophagy. PLoS One (2014) 9(6):e97957. doi: 10.1371/journal.pone.0097957

66. Fleischmajer R, Damiano V, Nedwich A. Alteration of subcutaneous tissue in systemic scleroderma. Arch Dermatol (1972) 105:59-66. doi: 10.1001/ archderm.105.1.59

67. Toldo S, Kannan H, Bussani R, Anzini M, Sonnino C, Sinagra G, et al. Formation of the inflammasome in acute myocarditis. Int J Cardiol (2014) Feb 15171(3):e119-21. doi: 10.1016/j.ijcard.2013.12.137

68. Abbate A. The heart on fire: inflammasome and cardiomyopathy. Exp Physiol (2013) Feb98(2):385. doi: 10.1113/expphysiol.2012.069021

69. Van Tassell BW, Toldo S, Mezzaroma E, Abbate A. Targeting interleukin-1 in heart disease. Circulation (2013) 128(17):1910-23. doi: 10.1161/ CIRCULATIONAHA.113.003199

70. Chung MK, Gulick TS, Rotondo RE, Schreiner GF, Lange LG. Mechanism of cytokine inhibition of beta-adrenergic agonist stimulation of cyclic AMP in rat cardiac myocytes. Impairment Signal transduction Circ Res (1990) Sep67 (3):753-63. doi: 10.1161/01.res.67.3.753

71. Liu SJ, Zhou W, Kennedy RH. Suppression of beta-adrenergic responsiveness of L-type Ca2+ current by IL-1beta in rat ventricular myocytes. Am J Physiol (1999) 276(1):H141-8. doi: 10.1152/ ajpheart.1999.276.1.H141

72. Schreur KD, Liu S. Involvement of ceramide in inhibitory effect of IL-1 beta on L-type Ca2+ current in adult rat ventricular myocytes. Am J Physiol (1997) 272(6 Pt 2):H2591-8. doi: 10.1152/ajpheart.1997.272.6.H2591 
73. Liu S, Schreur KD. G protein-mediated suppression of L-type Ca2+ current by interleukin-1 beta in cultured rat ventricular myocytes. Am J Physiol (1995) 268:C339-49. doi: 10.1152/ajpcell.1995.268.2.C339

74. Combes A, Frye CS, Lemster BH, Brooks SS, Watkins SC, Feldman AM, et al. Chronic exposure to interleukin lbeta induces a delayed and reversible alteration in excitation-contraction coupling of cultured cardiomyocytes. Pflugers Arch (2002) Nov445(2):246-56. doi: 10.1007/s00424-002-0921-y

75. McTiernan CF, Lemster BH, Frye C, Brooks S, Combes A, Feldman AM. Interleukin-1 beta inhibits phospholamban gene expression in cultured cardiomyocytes. Circ Res (1997) Oct81(4):493-503. doi: 10.1161/ 01.res.81.4.493

76. Tatsumi T, Matoba S, Kawahara A, Keira N, Shiraishi J, Akashi K, et al. Cytokine-induced nitric oxide production inhibits mitochondrial energy production and impairs contractile function in rat cardiac myocytes. $J \mathrm{Am}$ Coll Cardiol (2000) Apr35(5):1338-46. doi: 10.1016/s0735-1097(00) 00526-x

77. Schulz R, Panas DL, Catena R, Moncada S, Olley PM, Lopaschuk GD. The role of nitric oxide in cardiac depression induced by interleukin-1 beta and tumour necrosis factor-alpha. Br J Pharmacol (1995) 114(1):27-34. doi: 10.1111/j.1476-5381.1995.tb14901.x

78. Tsujino M, Hirata Y, Imai T, Kanno K, Eguchi S, Ito H, et al. Induction of nitric oxide synthase gene by interleukin-1 beta in cultured rat cardiocytes. Circulation. (1994) Jul90(1):375-83. doi: 10.1161/01.cir.90.1.375

79. Van Tassell BW, Arena RA, Toldo S, Mezzaroma E, Azam T, Seropian IM, et al. Enhanced interleukin-1 activity contributes to exercise intolerance in patients with systolic heart failure. PloS One (2012) 7(3):e33438. doi: 10.1371/journal.pone.0033438

80. Toldo S, Mezzaroma E, O'Brien L, Marchetti C, Seropian IM, Voelkel NF, et al. Interleukin-18 mediates interleukin-1-induced cardiac dysfunction. Am J Physiol Heart Circ Physiol (2014) 306(7):H1025-31. doi: 10.1152/ ajpheart.00795.2013

81. Toldo S, Mezzaroma E, Bressi E, Marchetti C, Carbone S, Sonnino C, et al. Interleukin-1 $\beta$ blockade improves left ventricular systolic/ diastolic function and restores contractility reserve in severe ischemic cardiomyopathy in the mouse. J Cardiovasc Pharmacol (2014) 64(1):1-6. doi: 10.1097/ FJC.0000000000000106

82. Kumar A, Thota V, Dee L, Olson J, Uretz E, Parrillo JE. Tumor necrosis factor alpha and interleukin 1beta are responsible for in vitro myocardial cell depression induced by human septic shock serum. J Exp Med (1996) Mar 1183(3):949-58. doi: 10.1084/jem.183.3.949

83. Lane JR, Neumann DA, Lafond-Walker A, Herskowitz A, Rose NR. Role of IL-1 and tumor necrosis factor in coxsackie virus-induced autoimmune myocarditis. J Immunol (1993) 151:1682-90.

84. Shioi T, Matsumori A, Sasayama S. Persistent expression of cytokine in the chronic stage of viral myocarditis in mice. Circulation. (1996) Dec 194 (11):2930-7. doi: 10.1161/01.cir.94.11.2930

85. Han RO, Ray PE, Baughman KL, Feldman AM. Detection of interleukin and interleukin-receptor mRNA in human heart by polymerase chain reaction. Biochem Biophys Res Commun (1991) Dec 16181(2):520-3. doi: 10.1016/ 0006-291x(91)91219-3

86. Vanderheyden M, Paulus WJ, Voss M, Knuefermann P, Sivasubramanian N, Mann D, et al. Myocardial cytokine gene expression is higher in aortic stenosis than in idiopathic dilated cardiomyopathy. Heart (2005) 91:926-31. doi: 10.1136/hrt.2004.035733

87. Eriksson U, Kurrer MO, Sonderegger I, Iezzi G, Tafuri A, Hunziker L, et al. Activation of dendritic cells through the interleukin 1 receptor 1 is critical for the induction of autoimmune myocarditis. J Exp Med (2003) 197:323-31. doi: $10.1084 /$ jem. 20021788

88. Lim BK, Choe SC, Shin JO, Ho SH, Kim JM, Yu SS, et al. Local expression of interleukin-1 receptor antagonist by plasmid DNA improves mortality and decreases myocardial inflammation in experimental coxsackieviral myocarditis. Circulation. (2002) Mar 19105(11):1278-81. doi: 10.1161/ circ.105.11.1278

89. Liu H, Hanawa H, Yoshida T, Elnaggar R, Hayashi M, Watanabe R, et al. Effect of hydrodynamics-based gene delivery of plasmid DNA encoding interleukin-1 receptor antagonist-Ig for treatment of rat autoimmune myocarditis: possible mechanism for lymphocytes and noncardiac cells. Circulation (2005) 111:1593-600. doi: 10.1161/01.CIR.0000160348.75918.CA
90. Tsutamoto T, Hisanaga T, Wada A, Maeda K, Ohnishi M, Fukai D, et al. Interleukin-6 spillover in the peripheral circulation increases with the severity of heart failure, and the high plasma level of interleukin-6 is an important prognostic predictor in patients with congestive heart failure. J Am Coll Cardiol (1998) Feb31(2):391-8. doi: 10.1016/s0735-1097(97)00494-4

91. Savvatis K, Müller I, Fröhlich M, Pappritz K, Zietsch C, Hamdani N, et al. Interleukin- 6 receptor inhibition modulates the immune reaction and restores titin phosphorylation in experimental myocarditis. Basic Res Cardiol (2014) 109(6):449. doi: 10.1007/s00395-014-0449-2

92. Campochiaro C, De Luca G, Tomelleri A, Sartorelli S, Peretto G, Sala S, et al. Tocilizumab for the Treatment of Myocardial Inflammation Shown by Cardiac Magnetic Resonance: Report of Two Cases and Rationale for Its Therapeutic Use. J Clin Rheumatol (2019). doi: 10.1097/ RHU.0000000000001194

93. Alivernini S, Gremese E, McSharry C, Tolusso B, Ferraccioli G, McInnes IB, et al. MicroRNA-155-at the Critical Interface of Innate and Adaptive Immunity in Arthritis. Front Immunol (2018) Jan 58:1932. doi: 10.3389/ fimmu.2017.01932

94. Park MJ, Moon SJ, Lee EJ, Jung KA, Kim EK, Kim DS, et al. IL-1-IL-17 Signaling Axis Contributes to Fibrosis and Inflammation in Two Different Murine Models of Systemic Sclerosis. Front Immunol (2018) 9:1611. doi: 10.3389/fimmu.2018.01611

95. Baldeviano GC, Barin JG, Talor MV, Srinivasan S, Bedja D, Zheng D, et al. Interleukin-17A is dispensable for myocarditis but essential for the progression to dilated cardiomyopathy. Circ Res (2010) May 28106 (10):1646-55. doi: 10.1161/CIRCRESAHA.109.213157

96. Liu Y, Zhu H, Su Z, Sun C, Yin J, Yuan H, et al. IL-17 contributes to cardiac fibrosis following experimental autoimmune myocarditis by a PKC $\beta / E r k 1 / 2 /$

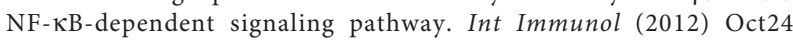
(10):605-12. doi: 10.1093/intimm/dxs056

97. Mantero JC, Kishore N, Ziemek J, Stifano G, Zammitti C, Khanna D, et al. Randomised, double-blind, placebo-controlled trial of IL1-trap, rilonacept, in systemic sclerosis. A phase I/II biomarker trial. Clin Exp Rheumatol (2018) 36 Suppl 1134):146-9.

98. Gasse P, Mary C, Guenon I, Noulin N, Charron S, Schnyder-Candrian S, et al. IL-1R1/MyD88 signaling and the inflammasome are essential in pulmonary inflammation and fibrosis in mice. J Clin Invest (2007) 117 (12):3786-99. doi: 10.1172/JCI32285

99. Cavalli G, Fallanca F, Dinarello CA, Dagna L. Treating pulmonary silicosis by blocking interleukin 1. Am J Respir Crit Care Med (2015) 191(5):596-8. doi: 10.1164/rccm.201412-2150LE

100. Cavalli G, Larcher A, Tomelleri A, Campochiaro C, Della-Torre E, De Luca $\mathrm{G}$, et al. Interleukin-1 and interleukin-6 inhibition compared with standard management in patients with COVID-19 and hyperinflammation: a cohort study. Lancet Rheumatol (2020). doi: 10.1016/S2665-9913(21)00012-6

101. Birnhuber A, Crnkovic S, Biasin V, Marsh LM, Odler B, Sahu-Osen A, et al. IL-1 receptor blockade skews inflammation towards Th2 in a mouse model of systemic sclerosis. Eur Respir J (2019) 2954(3):1900154. doi: 10.1183/ 13993003.00154-2019

102. Van Tassell BW, Abouzaki NA, Oddi Erdle C, Carbone S, Trankle CR, Melchior RD, et al. Interleukin-1 blockade in acute decompensated heart failure: a randomized, double-blinded, Placebo-Controlled Pilot Study. J Cardiovasc Pharmacol (2016) 67(6):544-51. doi: 10.1097/FJC.000000 0000000378

103. Van Tassell BW, Trankle CR, Canada JM, Carbone S, Buckley L, Kadariya D, et al. IL-1 Blockade in Patients With Heart Failure With Preserved Ejection Fraction. Circ Heart Fail (2018) 11(8):e005036. doi: 10.1161/ CIRCHEARTFAILURE.118.005036

104. Ridker PM, Everett BM, Thuren T, MacFadyen JG, Chang WH, Ballantyne C, et al. Antiinflammatory Therapy with Canakinumab for Atherosclerotic Disease. N Engl J Med (2017) 21377(12):1119-31. doi: 10.1056/ NEJMoa1707914

105. De Luca G, Campochiaro C, Dinarello CA, Dagna L, Cavalli G. Treatment of Dilated Cardiomyopathy With Interleukin-1 Inhibition. Ann Intern Med (2018) 169(11):819-20.. doi: 10.7326/L18-0315

106. Cavalli G, Franchini S, Aiello P, Guglielmi B, Berti A, Campochiaro C, et al. Efficacy and safety of biological agents in adult-onset Still's disease. Scand J Rheumatol (2015) 44(4):309-14. doi: 10.3109/03009742.2014.992949 
107. Cavalli G, De Luca G, Dagna L. Advances in potential targeted therapies for Erdheim-Chester disease. Expert Opin Orphan Drugs (2017) 5(3):253-60. doi: 10.1080/21678707.2017.1285226

108. De Luca G, Campochiaro C, Sartorelli S, Peretto G, Dagna L. Therapeutic strategies for virus-negative myocarditis: a comprehensive review. Eur J Intern Med (2020) 77:9-17. doi: 10.1016/j.ejim.2020.04.050

109. Vassilopoulos D, Lazaros G, Tsioufis C, Vasileiou P, Stefanadis C, Pectasides D. Successful treatment of adult patients with idiopathic recurrent pericarditis with an interleukin-1 receptor antagonist (anakinra). Int J Cardiol (2012) Sep 20160(1):66-8. doi: 10.1016/ j.ijcard.2012.05.086

110. Tomelleri A, Cavalli G, De Luca G, Campochiaro C, D’Aliberti T, Tresoldi $\mathrm{M}$, et al. Treating Heart Inflammation With Interleukin-1 Blockade in a Case of Erdheim-Chester Disease. Front Immunol (2018) 1(9):1233. doi: 10.3389/ fimmu.2018.01233

111. Cavalli G, Tomelleri A, De Luca G, Campochiaro C, Dinarello CA, Baldissera $\mathrm{E}$, et al. Efficacy of canakinumab as first-line biologic agent in adult-onset Still's disease. Arthritis Res Ther (2019) 1321(1):54. doi: 10.1186/s13075-019$1843-9$

112. Imazio M, Andreis A, De Ferrari GM, Cremer PC, Mardigyan V, Maestroni $\mathrm{S}$, et al. Anakinra for corticosteroid-dependent and colchicine-resistant pericarditis: The IRAP (International Registry of Anakinra for Pericarditis) study. Eur J Prev Cardiol (2019) Oct15:2047487319879534. doi: 10.1177/ 2047487319879534
113. Brucato A, Imazio M, Gattorno M, Lazaros G, Maestroni S, Carraro M, et al. Effect of Anakinra on Recurrent Pericarditis Among Patients With Colchicine Resistance and Corticosteroid Dependence: The AIRTRIP Randomized Clinical Trial. JAMA. (2016) Nov 8316(18):1906-12. doi: 10.1001/jama.2016.15826

114. Klein AL, Imazio M, Cremer P, Brucato A, Abbate A, Fang F, et al. Phase 3 Trial of Interleukin-1 Trap Rilonacept in Recurrent Pericarditis. N Engl J Med. (2021) 384(1):31-41. doi: 10.1056/NEJMoa2027892

115. Buckley LF, Libby P. Inhibiting NLRP3 inflammasome activity in acute myocardial infarction: a review of pharmacologic agents and clinical outcomes. J Cardiovasc Pharmacol (2019) 74:297-305. doi: 10.1097/ FJC.0000000000000701

Conflict of Interest: The authors declare that the research was conducted in the absence of any commercial or financial relationships that could be construed as a potential conflict of interest.

Copyright $\odot 2021$ De Luca, Cavalli, Campochiaro, Bruni, Tomelleri, Dagna and Matucci-Cerinic. This is an open-access article distributed under the terms of the Creative Commons Attribution License (CC BY). The use, distribution or reproduction in other forums is permitted, provided the original author(s) and the copyright owner(s) are credited and that the original publication in this journal is cited, in accordance with accepted academic practice. No use, distribution or reproduction is permitted which does not comply with these terms. 